\title{
Fixing cystic fibrosis CFTR with correctors and potentiators. Off to a good start
}

\section{J Stuart Elborn}

Since the cloning of the cystic fibrosis transmembrane regulator (CFTR) protein in the late 1980s and subsequent significant advances in understanding the pathophysiology of cystic fibrosis (CF) there have been high expectations that transformative, disease-modifying therapies could be developed. ${ }^{12}$ Dysfunction of the CFTR protein results in significant reduction or absence of chloride transport and dysregulation of sodium transport. ${ }^{3-5}$ Other cationic ions such as bicarbonate may also be transported by CFTR and the interaction with the epithelial sodium channel ENaC still remains to be elucidated. ${ }^{67}$ However, a significant number of in vitro and animal studies strongly support the premise that correction of CFTR function is possible and will make a significant impact on the CF phenotype, particularly in the lung, which accounts for most of the morbidity and mortality from this condition. ${ }^{7}$ Two approaches to correction of CFTR function are currently being pursued: one based on gene transfer therapy and the other on small molecule modulators.

Gene therapy attempts to correct CFTR dysfunction in CF by transfection of airway cells with the wild-type CFTR gene to enable cells to express functional CFTR. ${ }^{8}$ Progress with this approach has been slow and methodical. Early studies suggest that it is possible to transfect nasal and bronchial epithelial cells and this results in a modest electrophysiological improvement. ${ }^{9}{ }^{10}$ To date, there are no clinical studies in humans to determine if sufficient gene transfer can be achieved to result in a clinically meaningful benefit. A two-centre, double-blind, placebocontrolled trial of CF gene therapy is due to commence in the UK in 2012 using a specifically designed plasmid and a GL67 liposome complex. The results of this

Correspondence to Professor J Stuart Elborn, Professor of Respiratory Medicine, QUB and, Director of Centre for Infection and Immunity, Queen's University, Health Sciences Building, 97 Lisburn Road, Belfast, BT9 7BL, UK; s.elborn@qub.ac.uk proof of concept study will help determine if gene therapy has the potential to provide a treatment which will benefit all mutation classes in CF.

The second approach to modifying the underlying defect in CF has been the use of small molecules. A number of distinct approaches have been elucidated. The most straightforward is to use molecules that potentiate dysfunctional CFTR which reaches the cell membrane in patients with specific mutations associated with abnormal gating. ${ }^{11}$ These mutations, classified as class III mutations, account for around $5 \%$ of mutant alleles in people with $\mathrm{CF}^{12}$ The most frequently occurring of these gating mutations is G551D. ${ }^{12} 13$ In two recently published studies, a proof of concept ${ }^{14}$ and subsequent phase III study ${ }^{15}$ in patients with at least one G551D mutation, the potentiator VX-770 (Vertex Pharmaceuticals, Cambridge, Massachusetts, USA) was shown to have significant positive effects on nasal electrophysiology and sweat chloride concentrations. These effects translated into clinically relevant and sustained improvements in lung function, quality of life scores and body mass index, and significantly reduced pulmonary exacerbations. The improvements in lung function are of the highest magnitude seen for any therapy in a randomised controlled trial of patients with CF. These findings suggest that correction of the chloride defect indicated by a $50 \%$ reduction in sweat chloride concentration translates into a $10 \%$ improvement in forced expiratory volume in $1 \mathrm{~s}$. In addition to a substantial reduction in pulmonary exacerbations, this strongly suggests that VX-770 in patients with G551D is a disease-modifying therapy. Other gating mutations are less common in $\mathrm{CF}$ and account for $<1 \%$ of mutant alleles. In vitro studies using CF epithelial cell lines show that the gating mutations respond to VX-770 in a similar manner to the G551D mutation in vitro. There are no clinical data on patients with these mutations but there is no reason to anticipate a different clinical response from patients with G551D. There are too few patients in the world with these mutations to allow conventional clinical trials to be conducted. VX-770 may also potentiate and improve the function of class IV mutations which have conductance defects. The most common of these mutations is the R117H mutation, which when found in cis with a 5T splice variant (IVS8-T5) and a second severe CF mutation results in classic CF. ${ }^{13}$ Further studies are required in this larger patient group. Correcting the most common CFTR mutation F508del will however be more challenging.

F508del is primarily a class II mutation and results from misfolding of mutant CFTR, which is mostly degraded intracellularly in the proteasome and so does not reach the cell membrane. ${ }^{16}$ However, in vitro observations using cooled cells and compounds which overcome the proteasomal disposal show that it is possible to make F508del bypass the proteasomal degradation system, resulting in it being expressed at the cell membrane. ${ }^{16-18}$ At the cell membrane F508del then behaves as a gating mutation similar to G551D and can be activated with correctors such as VX-770. ${ }^{15}$ A number of in vitro compounds have been shown to have this effect, so correcting the F508del mutation. In this issue of Thorax the first significant clinical study of a pharmaceutical corrector demonstrates that in vivo treatment with VX-809, a CFTR modulator, results in a dose-dependent reduction in sweat chloride concentrations. ${ }^{19}$ In this well designed study, safety was the primary outcome but secondary outcome measures similar to the successful studies with VX-770 were also investigated. These included sweat chloride concentration, nasal potential difference, measures of lung function and patient symptoms. No safety concerns were demonstrated and encouragingly modest but significant, dose-dependent reductions in sweat chloride were demonstrated in treated patients compared with those receiving placebo. The reduction in the group treated with the highest dose of VX-809 was $8.0 \mathrm{mmol} /$ litre. This compares to a much larger reduction in sweat chloride concentration of around $60 \mathrm{mmol} /$ litre in patients with G551D treated with VX-770. ${ }^{15}$ No significant changes were seen in CFTR function using nasal potential difference or maturation of CFTR protein and no changes were demonstrated in lung function or 
patient-reported outcomes. However, the study was not powered to determine these outcomes as it was primarily a safety study.

Although the magnitude of the effect of VX-890 on sweat chloride concentrations in people with CF homozygous for the F508del CFTR mutation is considerably lower than the impact of the potentiator VX-770 in patients with G551D mutations, the data from this study are encouraging as they demonstrate that it is possible to have an electrophysiological effect following correction of F508del CFTR. In this study there are no supportive data that VX-809 changed the amount of CFTR at the cell membrane, but the changes in chloride concentration suggest this is the likely mechanism. F508del CFTR at the cell membrane behaves as a gating mutation and can be potentiated by VX-770. The next question therefore is if combined with VX-770 in F508del homozygous patients, will there be a greater effect on sweat chloride concentrations and will this be sufficient to result in a clinical benefit? Phase II studies are currently underway to help determine this. As yet we do not know how much change in sweat chloride is required to result in an associated clinical benefit. This will be difficult to determine because the two are not directly linked, but it is an intriguing possibility that sweat testing may be a way of monitoring early response to drugs that modulate CFTR function.

These studies and others soon to be reported represent a watershed in how we view the treatment of $C F$. The studies with VX-770 in patients with G551D indicate that the CFTR mutant protein is tractable with a small molecule drug and this translates into important and almost certainly disease-modifying improvements in people with CF and a gating mutation. The current study in Thorax gives significant encouragement that the more common mutation F508del may also be tractable and further studies using the combination of VX-809 and VX-770 and other CFTR modulators (correctors and potentiators) are underway and results are eagerly anticipated.

A further small molecule, PTC124 (Ataluren, PTC Therapeutics, South Plainfield, New Jersey, USA), promotes read through in stop codon mutations in patients with class I mutations. In early proof-of-concept studies lung function responses are less than those seen in the VX-770 study but are still encouraging. ${ }^{20} 21$
A multicentre pivotal trial will report soon.

The clinical trials of these diseasemodifying approaches in the next 3 years may change how we view therapy in CF. Modifying disease progression in patients with an already established phenotype will be important but early intervention studies may offer even more significant long-term benefits for people with CF. Studies in infants and children will be challenging but are a key future focus for clinical trials. So far, VX770 and VX809 have shown no major concerning side effects. The number of patients exposed to these drugs is still relatively small and careful post-licensing surveillance will be important, particularly for VX770 which may be available in 2012 .

The research programmes for these two compounds from Vertex Pharmaceuticals are also to be commended. The development programme for VX-770 and VX-809 was supported by the Cystic Fibrosis Foundation (USA) with Vertex and demonstrates that the charitable sector can work effectively with industry in orphan diseases to progress important new therapies that may not be financially viable for small and medium sized companies working alone. The clinical trials of VX-770 and VX-809 demonstrate a wide international collaboration involving North America, Europe and Australasia working closely together to ensure these clinical trials are delivered as quickly as possible despite the relatively small number of patients, particularly with G551D mutations. This is an important development in delivery of high-quality, efficiently executed clinical trials in an orphan disease such as CF. The next 5 years in the care of patients with CF will be exciting and challenging. These studies suggest we will have new and effective tools to treat the basic defect in $\mathrm{CF}$ and so potentially prevent disease developing, and to modify the phenotype in patients with already established disease.

Competing interests JSE has received consultancy fees from Vertex and was CO P1 of Vx-102 study. ${ }^{15}$

Provenance and peer review Commissioned; internally peer reviewed.

Published Online First 5 November 2011

Thorax 2012;67:4-5.

doi:10.1136/thoraxjnl-2011-201197

\section{REFERENCES}

1. Riordan JR, Rommens JM, Kerem B, et al. Identification of the cystic fibrosis gene: cloning and characterization of complementary DNA. Science 1989;245:1066-73.

2. Berger HA, Anderson MP, Gregory RJ, et al. Identification and regulation of the cystic fibrosis transmembrane conductance regulator-generated chloride channel. J Clin Invest 1991;88:1422-31.

3. Knowles M, Gatzy J, Boucher R. Relative ion permeability of normal and cystic fibrosis nasal epithelium. J Clin Invest 1983;71:1410-17.

4. Quinton PM. Chloride impermeability in cystic fibrosis. Nature 1983;301:421-2.

5. Welsh MJ, Smith AE. Molecular mechanisms of CFTR chloride channel dysfunction in cystic fibrosis. Cell 1993;73:1251-4.

6. Poulsen JH, Fischer $\mathrm{H}$, Illek B, et al. Bicarbonate conductance and $\mathrm{pH}$ regulatory capability of cystic fibrosis transmembrane conductance regulator. Proc Natl Acad Sci U S A 1994;91:5340-4.

7. Mall M, Bleich M, Greger R, et al. The amilorideinhibitable $\mathrm{Na}+$ conductance is reduced by the cystic fibrosis transmembrane conductance regulator in normal but not in cystic fibrosis airways. J Clin Invest 1998;102:15-21.

8. Alton E, Ferrari S, Griesenbach U. Progress and prospects: gene therapy clinical trials (part 1). Gene Ther 2007:14:1439-47.

9. Alexander BL, Ali RR, Alton EW, et al. Progress and prospects: gene therapy clinical trials (part 1). Gene Ther 2007:14:1754.

10. McLachlan G, Davidson D, Holder E, et al. Pre-clinical evaluation of three non-viral gene transfer agents for cystic fibrosis after aerosol delivery to the ovine lung. Gene Ther 2011;18:996-1005.

11. Van Goor F, Hadida S, Grootenhuis PD, et al. Rescue of CF airway epithelial cell function in vitro by a CFTR potentiator, VX-770. Proc Natl Acad Sci U S A 2009; 106:18825-30.

12. The Molecular Genetic Epidemiology of Cystic Fibrosis. Report of a Joint Meeting WHO/ECFTN/ICF (M)A/ECFS. Geneva: WHO Human Genetics Programme WHO/HGN/CF/WG/04.02.

13. Comer DM, Ennis M, McDowell C, et al. Clinical phenotype of cystic fibrosis patients with the G551D mutation. OJM 2009;102:793-8.

14. Accurso FJ, Rose SM, Clancy JP. Effect of VX-770 in persons with cystic fibrosis and the G551DCFTR mutation. N Engl J Med 2010;363:1991-2003.

15. Ramsey BW, Davies J, McElvaney NG, et al. A CFTR potentiator in patients with cystic fibrosis who have the G551D mutation. N Engl J Med 2011:365:1663-72.

16. Yu H, Burton B, van Goor F. VX-770 an investigationa CFTR potentiator acts on multiple CFTR forms in vitro. Ped Pulmonol 2010;(Suppl 33):318-19.

17. Cheng SH, Gregory RJ, Marshall J, et al. Defective intracellular transport and processing of CFTR is the molecular basis of most cystic fibrosis. Cell 1990;63:827-34.

18. Denning GM, Anderson MP, Amara JF, et al. Processing of mutant cystic fibrosis transmembrane conductance regulator is temperature-sensitive. Nature 1992;358:761-4.

19. Clancy JP, Rowe SM, Accurso FJ, et al. Results of a phase 2a study of VX-809, an investigational CFTR corrector compound, in cystic fibrosis subjects homozygous for the F508del-CFTR mutation. Thorax 2012;67:12-8.

20. Sermet-Gaudelus I, De Boeck K, Casimir GJ, et al Ataluren (PTC124) induces cystic fibrosis transmembrane conductance regulator protein expression and activity in children with nonsense mutation cystic fibrosis. Am J Respir Crit Care Med 2010;182:1262-72.

21. Wilskanski M, Kerem E. New drugs for cystic fibrosis. Expert Opin Investig Drugs 2011:21:4824-31. 